\title{
Cis-2-dodecenoic acid signal modulates virulence of Pseudomonas aeruginosa through interference with quorum sensing systems and T3SS
}

\author{
Yinyue Deng ${ }^{1,2^{*}}$, Calvin Boon ${ }^{1}$, Shaohua Chen ${ }^{2}$, Amy Lim ${ }^{1}$ and Lian-Hui Zhang ${ }^{1,2^{*}}$
}

\begin{abstract}
Background: Cis-2-dodecenoic acid (BDSF) is well known for its important functions in intraspecies signaling in Burkholderia cenocepacia. Previous work has also established an important role of BDSF in interspecies and inter-kingdom communications. It was identified that BDSF modulates virulence of Pseudomonas aeruginosa. However, how BDSF interferes with virulence of $P$. aeruginosa is still not clear.

Results: We report here that BDSF mediates the cross-talk between $B$. cenocepacia and $P$. aeruginosa through interference with quorum sensing (QS) systems and type III secretion system (T3SS) of $P$. aeruginosa. Bioassay results revealed that exogenous addition of BDSF not only reduced the transcriptional expression of the regulator encoding gene of QS systems, i.e., lasR, pqsR, and $r h / R$, but also simultaneously decreased the production of QS signals including 3-oxo-C12-HSL, Pseudomonas quinolone signal (PQS) and C4-HSL, consequently resulting in the down-regulation of biofilm formation and virulence factor production of $P$. aeruginosa. Furthermore, BDSF and some of its derivatives are also capable of inhibiting T3SS of $P$. aeruginosa at a micromolar level. Treatment with BDSF obviously reduced the virulence of $P$. aeruginosa in both HeLa cell and zebrafish infection models.
\end{abstract}

Conclusions: These results depict that BDSF modulates virulence of $P$. aeruginosa through interference with QS systems and T3SS.

\section{Background}

Many microorganisms utilize species-specific small signal molecules to coordinate a range of important activities, including virulence factor production, antibiotics biosynthesis and biofilm. This cell-cell communication mechanism is known as quorum sensing (QS) [1-4]. As a ubiquitous environmental organism which infects various animals, plants, and insects, Pseudomonas aeruginosa is also a major source of opportunistic infections in both immunocompromised individuals and cystic fibrosis patients $[5,6]$. It has evolved at least three types of quorum sensing (QS) systems, i.e., las, $p q s$ and $r h l$, which are implicated in regulation of several aspects of pathogenesis, including virulence factor production, biofilm development, and

\footnotetext{
* Correspondence: ydeng@imcb.a-star.edu.sg; lianhui@imcb.a-star.edu.sg 'Institute of Molecular and Cell Biology, Proteos, 61 Biopolis Drive, Singapore, 138673, Singapore

${ }^{2}$ College of Natural Resources and Environment, South China Agricultural University, Guangzhou, People's Republic of China
}

antimicrobial resistance [7]. Besides the QS systems, most clinical isolates of $P$. aeruginosa also use type III secretion system (T3SS) to evade phagocytosis and facilitate infection [8-13]. T3SS is an important virulence determinant which is conserved in many animal and plant pathogens, including Salmonella spp., Shigella flexneri, Yersinia spp., Escherichia coli, and Chlamydia spp. [14,15]. Although previous study already revealed that QS controls T3SS in some bacterial species, the relationship between QS systems and T3SS in P. aeruginosa is still not clearly determined $[16,17]$.

There is a new form of microbe-microbe antagonism interaction designated as signal interference [18]. This type of antagonism acts not by killing, but instead by interfering with the signal-mediated gene expression of the competitors $[19,20]$. It has been found that some microorganisms could boost their competitive strength through interfering with QS signaling of their competitors [18]. This interference mechanism has been employed to develop novel

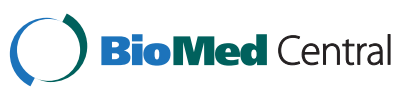


drugs as the antagonists of signaling systems of bacterial pathogens. For example, some compounds have been identified or synthesized to act as the antagonists of QS systems of $P$. aeruginosa, including $N$-acyl cyclopentylamides, Furanone derivatives, Garlic, Malyngolide, Iberin, Protoanemonin, Norbgugaine and Caffeine [21-28]. Besides the signal interference with QS system, interfering with T3SS system has also been shown as an effective method to treat the bacterial pathogens [29-31]. Given the fact that the anti-QS or anti-T3SS compounds display a significantly inhibitory activity on the virulence of $P$. aeruginosa, suggesting that interference on QS systems or/and T3SS can be specifically utilized as favorable therapeutic methods on P. aeruginosa infection [32-34].

As a new type of QS signal, the diffusible signal factor (DSF) has been demonstrated to play an important role to control the biological functions such as biofilm formation, motility, virulence and antibiotic resistance [3,4]. Among DSF-family signals, Cis-2-dodecenoic acid (BDSF) was originally identified in Burkholderia cenocepacia to be involved in regulation of biofilm formation, virulence and motility in B. cenocepacia [35-41]. Furthermore, besides the significance on intraspecies signaling, BDSF also appears its critical importance for maintenance of ecology through interspecies and inter-kingdom communication [35,37,42]. Exogenous addition of BDSF from B. cenocepacia restored the biofilm dispersal and virulence factor production of Xanthomonas campestris pv. campestris DSF-deficient mutants. It was also revealed that Candida albicans germ tube formation was strongly inhibited by exogenous addition of physiological relevant level of BDSF [35]. Moreover, BDSF from B. cenocepacia and Stenotrophomonas maltophilia was found to be involved in modulation of virulence, antibiotic resistance and persistence of $P$. aeruginos $a$ in the cystic fibrosis airway [42]. In combination, these results have well established the role of BDSF play not only in regulation of a range of biological functions through intraspecies signaling, but also in microbial ecology through interspecies and cross-kingdom communication. In this study, we investigate the role of BDSF in the inter-species communication between $B$. cenocepacia and $P$. aeruginosa; and discover the inhibitory effect of BDSF on QS systems and T3SS of $P$. aeruginosa.

\section{Results}

\section{BDSF interferes with QS systems of $P$. aeruginosa}

There are at least two AHL-dependent QS systems in $P$. aeruginosa, las and $r h l$ systems, which control the expression of numerous genes $[34,43,44]$. It was found that the two QS systems are in a hierarchy where las system is dominant over rhl system [45,46]. Moreover, it was also identified that LasR was required for the production of PQS, which plays a positive effect on $r h l$ system. The three QS systems are interconnected with las system at the top of the QS cascade, which controls $r h l$ and $p q s$ systems [47,48]. Recently, it was revealed that pqs and $r h l$ systems are under the positive regulation of iqs system (It was named for its role in integrating the QS network in $P$. aeruginosa), which is tightly controlled by las system in $P$. aeruginosa [49].

It was found that there is a regulatory interaction between the different type QS systems, BDSF and AHL systems in B. cenocepacia [39,40]. Disruption of both BDSF synthase and receptor caused a substantial downregulation of AHL signals production and AHL synthase gene expression in B. cenocepacia. Given the fact that $B$. cenocepacia and $P$. aeruginosa share the same niche in cystic fibrosis patent, we then hypothesized that BDSF may play a regulatory role in the QS systems of $P$. aeruginosa. To determine the influences of BDSF on the QS systems of $P$. aeruginosa, the promoter-lacZ fusion reporters were constructed to test the transcriptional expression of the regulator encoding gene of QS systems, i.e., lasR, pqsR and $r h l R$, in the absence and presence of BDSF. It was shown that exogenous addition of BDSF did not obviously affect the bacterial growth of $P$. aeruginosa PA14 (Figure 1A). But the transcriptional expressions of las $R$, $p q s R$ and $r h l R$ were repressed by treatment with BDSF in a dosage-dependent manner. Addition of $0.25 \mathrm{mM}$ BDSF resulted in 38\%, 35\% and 48\% reduction of the expression of lasR, pqsR and rhlR, respectively (Figure 1B-D). To further investigate BDSF effect on the QS signal production of $P$. aeruginosa PA14, production of 3-oxo-C12-HSL, PQS, and C4-HSL were tested in the absence and presence of BDSF. Consistently, it was revealed that treatment with BDSF decreased the production of 3-oxo-C12-HSL, PQS and C4-HSL. As shown in Additional file 1: Figure S1, addition of BDSF slightly decreased the production of 3-oxo-C12-HSL; while obviously inhibited the production of PQS and C4-HSL.

\section{BDSF inhibits biofilm formation and virulence factor production of $P$. aeruginosa}

It is well known that QS systems in P. aeruginosa modulate biofilm development, virulence factor production, and antimicrobial resistance [7]. Linking with the finding of the inhibitory activity of BDSF on the QS systems, we then continued to determine the effect of BDSF on the biofilm formation and virulence factor production. As shown in Figure 1A, exogenous addition of BDSF showed no obvious effect on the growth rate of $P$. aeruginosa PA14; while it remarkably decreased the biofilm formation. Addition of $0.05,0.1,0.25$ and $0.5 \mathrm{mM}$ BDSF reduced the biofilm formation by $10.2 \%, 20.2 \%, 27.9 \%$, and $44 . \%$, respectively (Figure 2A).

$P$. aeruginosa usually utilizes exoenzyme to induce its pathogenesis $[50,51]$. To study the effect of BDSF on the production of exoproteases, which are important virulence 

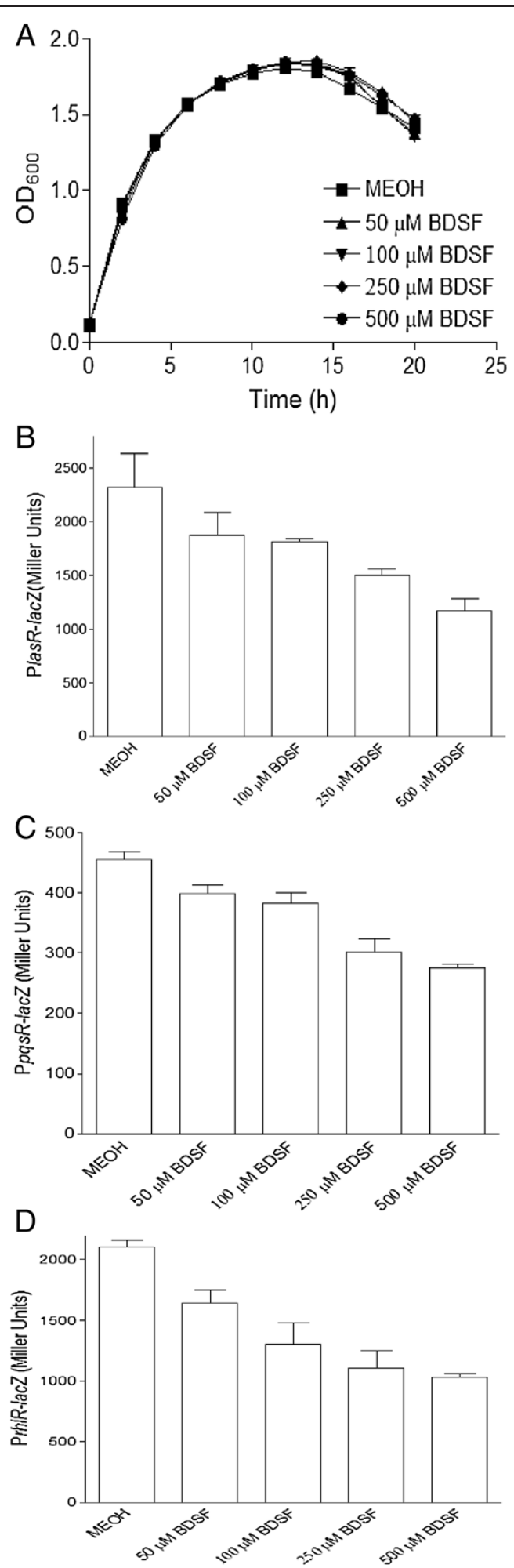

Figure 1 Influence of BDSF on QS systems of $P$. aeruginosa.

Effect of BDSF on the growth rate of $P$. aeruginosa PA14 (A), and on the transcriptional expression of las $R$ (B), pqs $R$ (C), and $r h / R$ (D), as determined by using corresponding promoter-lac $Z$ fusion reporter strains. The data are the means of three repeats and error bars indicate the standard deviations.

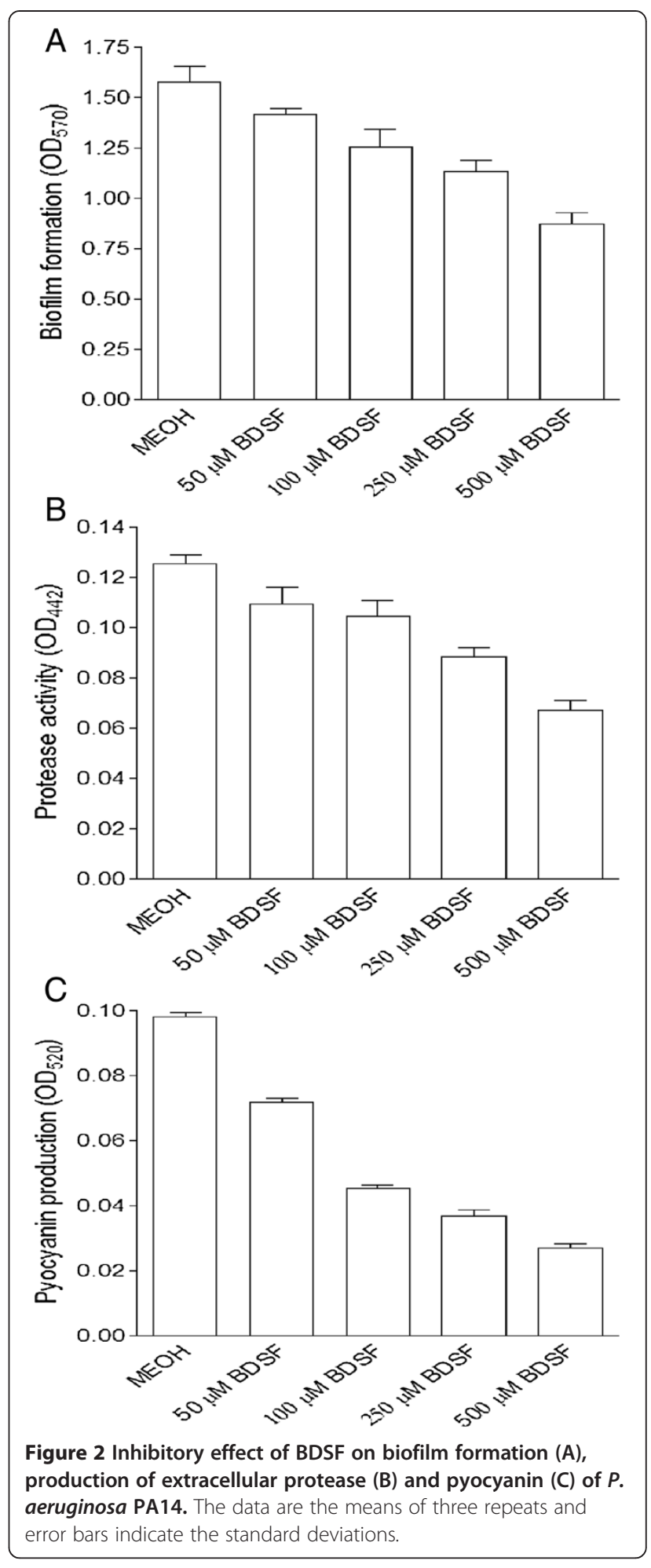

factors of $P$. aeruginosa, BDSF was added to the growth medium of $P$. aeruginosa PA14 to test its effect on the secretion of proteases in the supernatants. Results demonstrated that addition of BDSF inhibited the production of proteases, treatment with $0.25 \mathrm{mM}$ BDSF caused 30\% 
reduction of the protease activity (Figure $2 \mathrm{~B}$ ). The reduction was increased to $50 \%$ when $0.5 \mathrm{mM}$ BDSF was supplemented (Figure 2B).

During the growth process, it was observed that BDSF inhibited the pigment accumulation of $P$. aeruginosa PA14 in the medium. $P$. aeruginosa produces a number of colored secondary metabolities; one of them is pyocyanin, which is a virulence factor $[52,53]$. To determine whether BDSF affects this virulence factor production, we measured pyocyanin accumulation in LB medium in the absence and presence of BDSF. As shown in Figure 2C, addition of BDSF to the bacterial medium substantially reduced the production of pyocyanin. Compared with the control, addition of 0.1 and $0.5 \mathrm{mM}$ BDSF reduced the pyocyanin production by about $55 \%$ and $70 \%$, respectively (Figure $2 \mathrm{C}$ ).

It was reported that DSF from S. maltophilia influences biofilm formation and polymyxin tolerance in $P$. aeruginosa through the sensor kinase PA1396 [54]. To test whether PA1396 is also the sensor of BDSF in $P$. aeruginosa, we then measured the protease activity and pyocyanin production of PA1396 deletion mutant in the absence and presence of BDSF. However, our results showed that disruption of PA1396 was not able to diminish the inhibitory effect of BDSF on the virulence factor production (Additional file 1: Figure S2), suggesting that PA1396 is not BDSF sensor kinase in P. aeruginosa.

\section{Exogenous addition of BDSF represses T3SS of $P$. aeruginosa} Besides the QS systems, T3SS is also an important virulence determinant in $P$. aeruginosa. We firstly studied the effect of BDSF on T3SS of $P$. aeruginosa by using semiquantitative RT-PCR. At the panel of 5 ng RNA, results showed that addition of $100 \mu \mathrm{M}$ BDSF to $P$. aeruginosa led to about $30 \%$ and $50 \%$ reduction in the signal density of exs $C$ and exsA, which are the master regulators and positively control the expression of T3SS effectors genes in $P$. aeruginosa (Figure 3A) $[13,55,56]$. We then continued to measure the effect of BDSF on T3SS effectors. Semi-quantitative RT-PCR analysis showed that treatment of $P$. aeruginosa PA14 with $100 \mu \mathrm{M}$ BDSF caused about $39 \%$ and $17 \%$ reduction in transcripts levels of exoS and exoT at the panel of $50 \mathrm{ng}$ RNA, respectively (Figure 3A). Furthermore, western blotting assay was used to analyze the effect of BDSF on the secreted ExoS in supernatant. As shown in Figure 3B, addition of $100 \mu \mathrm{M}$ BDSF significantly reduced the amount of ExoS secreted in supernatant. When the final concentration of BDSF was increased to $500 \mu \mathrm{M}$, there was almost no detectable protein band of ExoS.

It was previously reported that a long-chain fatty acid (LCFA) negatively modulates the expression of type III exsCEBA operon in $P$. aeruginosa through the sensor PsrA [57]. To investigate whether BDSF shares the same

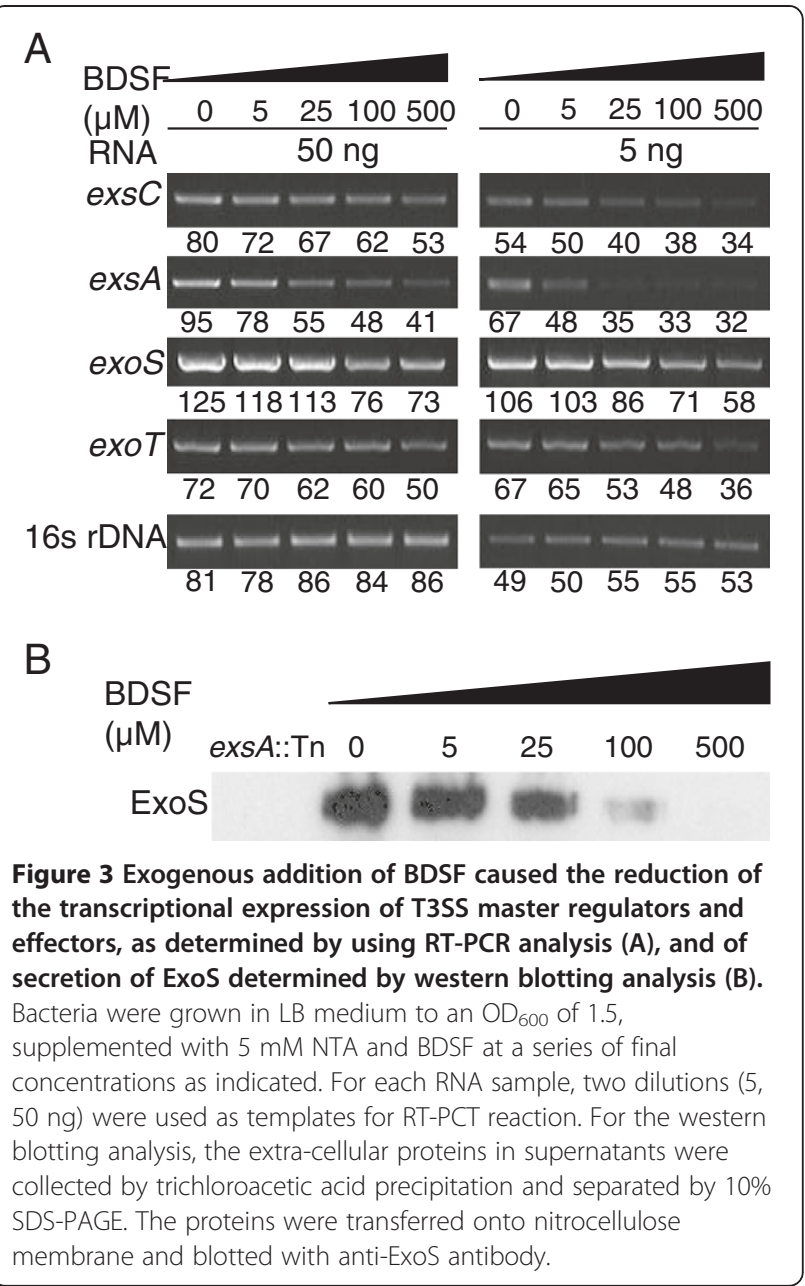

signaling pathway as LCFA, we then further measured the effect of BDSF on T3SS in $P$. aeruginosa psrA mutant. Unexpectedly, deletion of psrA displayed no effect on the inhibitory activity of BDSF on T3SS of $P$. aeruginosa, suggesting that BDSF possibly acts through another sensor different from that of the LCFA molecule (Additional file 1: Figure S3).

\section{BDSF inhibits T3SS of $P$. aeruginosa at its physiological relevant level}

To determine whether BDSF affects T3SS gene expression in $P$. aeruginosa PA14 at its physiological relevant level, T3SS reporter PexsCEBA-lacZ was used to test the T3SS gene expression at transcriptional level. B. cenocepacia cells were firstly grown in Luria Bertani (LB) broth to an $\mathrm{OD}_{600}$ of 3.5; and cultures were centrifuged to collect the supernatants, which were then filtered with $0.2 \mu \mathrm{m}$ syringe filter to remove the remaining cells. T3SS reporter strain was inoculated in the mixture of the supernatant of $B$. cenocepacia strains and LB broth at a ratio of 1:1 (v/v), with supplementation of $5 \mathrm{mM}$ nitrilotiracetic acid (NTA). The bioassay result showed that the reporter strain displayed the greatest 
activity when it was cultured in the supernatant of BDSFdeficient mutant; while the supernatants of both the wildtype strain and over-expression complementary strain showed a remarkable inhibition on the T3SS gene expression at the time points examined (Figure 4).

\section{Many BDSF derivatives show inhibitory activity on T3SS of $P$. aeruginosa}

It was revealed that structural features of fatty acids molecules may contribute to their biological activity $[4,37,58]$. To investigate whether BDSF derivatives share the inhibitory activity on T3SS of $P$. aeruginosa, and study the interaction between structural features and inhibitory activities of DSF-family molecules on T3SS, a series of BDSF derivatives with different structures (Additional file 1: Table S1) were applied to test their effect on the transcriptional expression of exsCEBA. The T3SS reporter strain, PexsCEBA-lacZ, was refreshed and cultured in LB medium supplemented with $5 \mathrm{mM}$ NTA. BDSF and its derivatives were added at a final concentration of $10 \mu \mathrm{M}$. Beta-gal activity was measured when the cultures reached an $\mathrm{OD}_{600}$ of $\sim 1.5$. Bioassays results revealed that $\alpha, \beta$-unsaturated fatty acids with chain length more than twelve showed a significant inhibition on T3SS gene expression (Figure 5). Moreover, it was indicated that configuration of BDSF derivatives contributes to their inhibitory activity; and cis-conformational fatty acids showed the strongest inhibitory activity, followed by their saturated isomers and trans-isomers (Figure 5B). Additionally, methyl group substitution and chain length could also affect the inhibitory activity (Figure 5A).

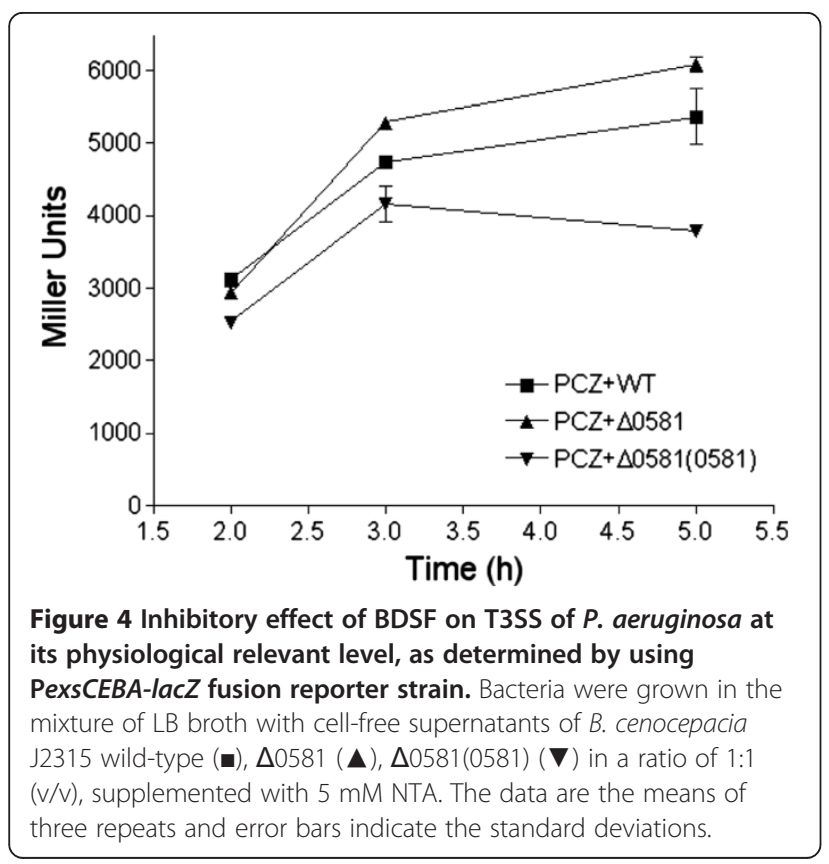

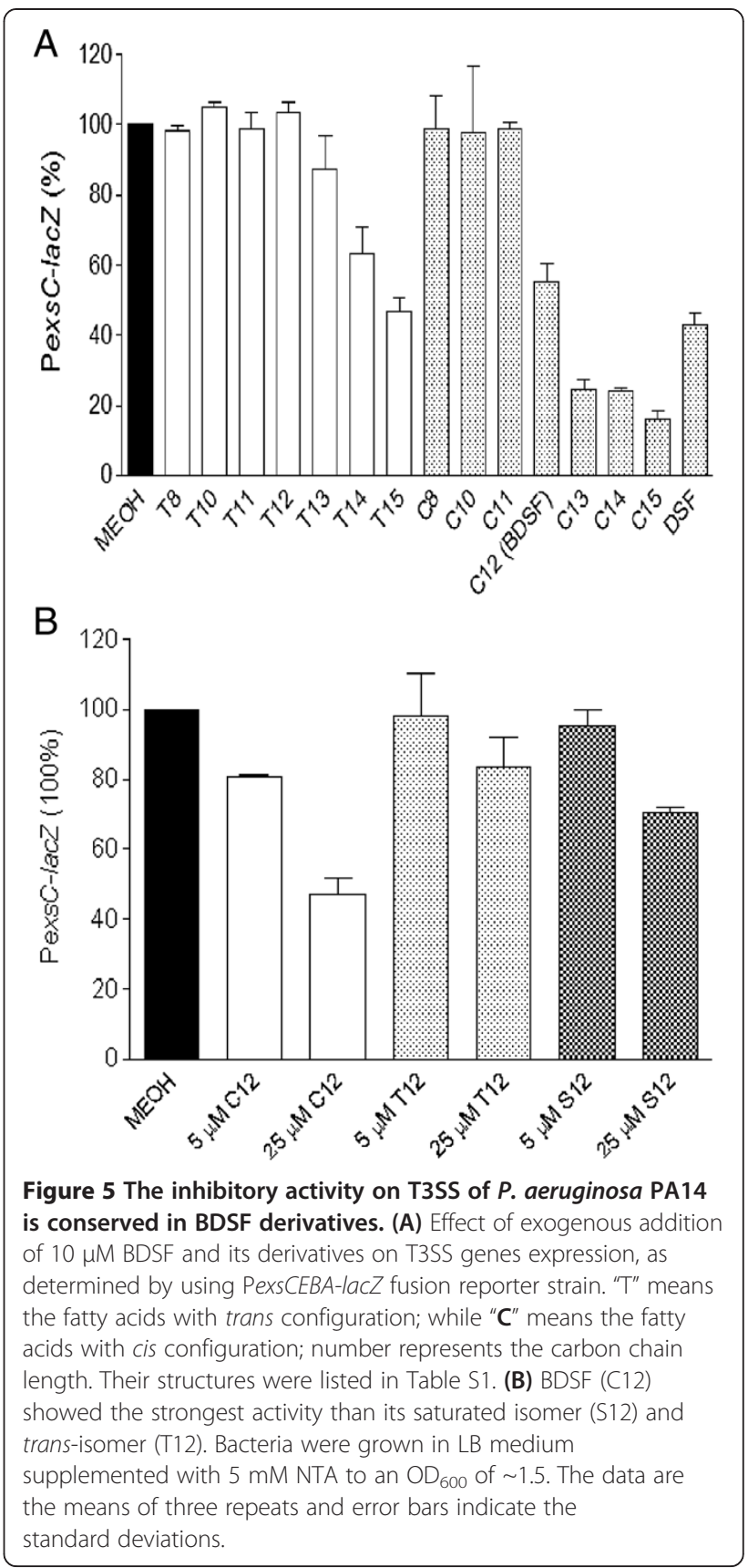

BDSF attenuates the virulence of $P$. aeruginosa in both in vitro and in vivo assays

To determine the effect of BDSF on the virulence of $P$. aeruginosa PA14, HeLa cells were firstly used as the in vitro model. Results indicated that exogenous addition of BDSF significantly decreased the cytotoxicity of $P$. aeruginosa PA14 to HeLa cell. For 2 hours inoculation, the cytotoxicity was reduced by $41 \%$ and $75 \%$ with treatment of $5 \mu \mathrm{M}$ and $25 \mu \mathrm{M}$ BDSF, respectively (Figure 6A). While for 5 hours inoculation, the reductions were 16\% and $73 \%$, respectively (Figure 6A). 


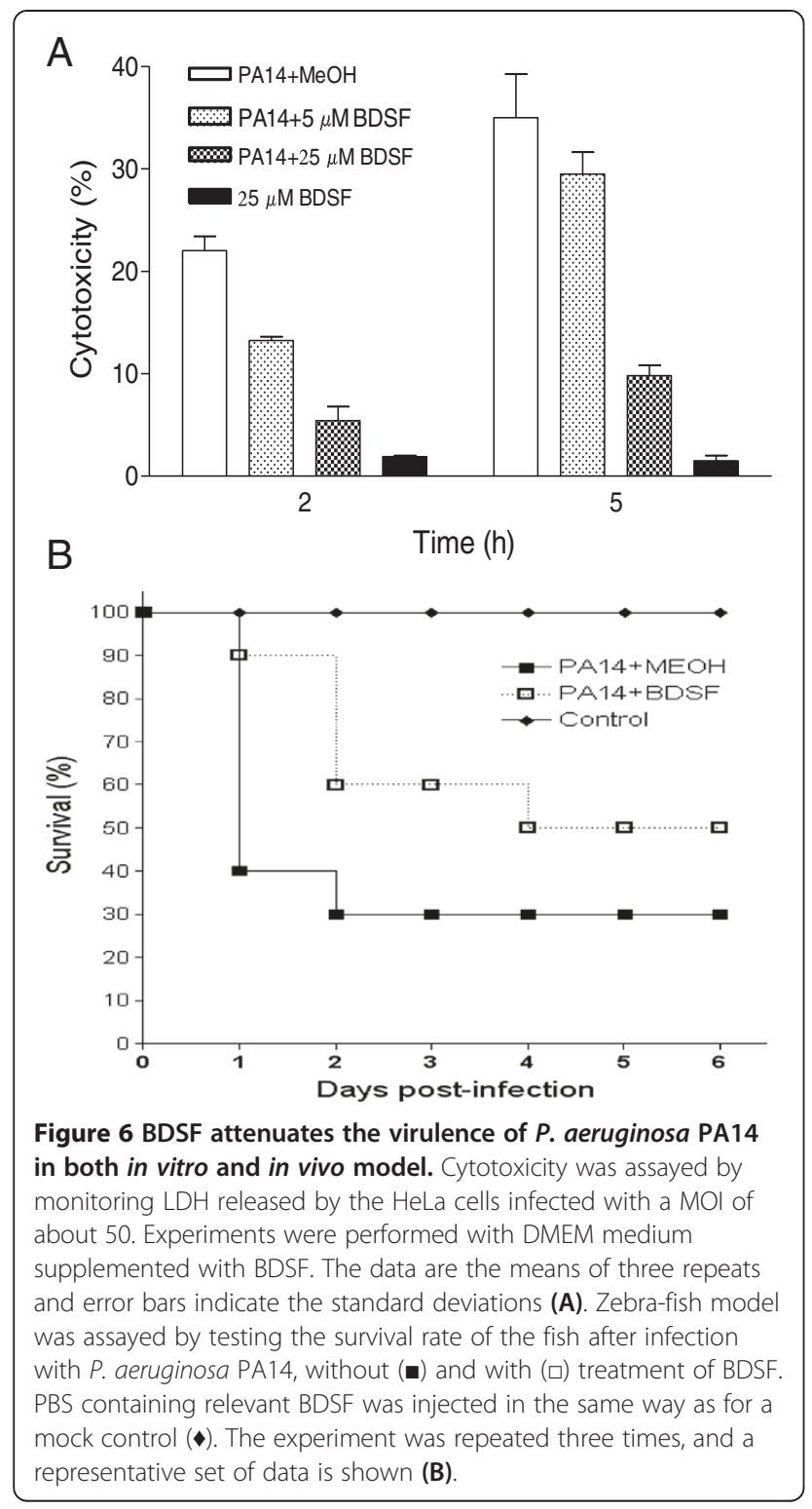

Zebrafish was reported to be a good infection model for the test of T3SS-mediated virulence of $P$. aeruginosa [59]. We then analyzed the effect of BDSF on the virulence of $P$. aeruginosa PA14 by infecting 6-month-old zebrafish (Danio rerio), which was used as an infection model for B. cenocepacia [36]. As shown in Figure 6B, treatment with $100 \mu \mathrm{M}$ BDSF significantly reduced the virulence of $P$. aeruginosa PA14 in zebrafish infection model. While $60 \%$ of the fish infected with $P$. aeruginosa PA14 died within 24 hours post-inoculation, the $P$. aeruginosa PA14 treated with BDSF was much less virulent and only $10 \%$ of the infected fish died (Figure 6B).

\section{Discussion}

This study demonstrated that besides acting as an intraspecies signal, BDSF can also mediate inter-species communication between $B$. cenocepacia and $P$. aeruginosa through inhibiting on QS systems and T3SS of $P$. aeruginosa. Previous studies already found that there is a cross-talk between B. cepacia and P. aeruginosa, which is modulated by AHL QS signal from P. aeruginosa [60,61]. It was reported that addition of cell-free exoproducts of PAO1 to B. cepacia markedly enhanced its production of siderophore, lipase and protease; while addition of the supernatants of PAO1 with attenuated production of AHL only had slight effect on the production of these virulence factors [60]. Moreover, AHL from PAO1 can also induce the biofilm formation of B. cenocepacia H111, demonstrating that AHL signal plays an important role in the cross-talk between the two bacterial species, which usually share the same niche in cystic fibrosis patients. Recently, the critical role of BDSF in the interspecies communication between $B$. cenocepacia and $P$. aeruginosa was also well established by the finding that BDSF modulates virulence, antibiotic resistance and persistence of $P$. aeruginosa in the cystic fibrosis airway [42]. Our findings not only further confirmed the BDSF role in the complicated interaction between B. cenocepacia and $P$. aeruginosa, but also depicted how BDSF modulates the virulence of $P$. aeruginosa.

In $P$. aeruginosa, there are at least three QS systems, i.e., las, $p q s$ and $r h l$, consisting of a hierarchy regulatory network. These QS systems regulate a broad range of genes important for the metabolism and virulence of $P$. aeruginosa. Inhibition on these QS systems will finally attenuate the virulence factor production and virulence of $P$. aeruginosa. Our study showed that addition of BDSF caused the decreased expression of lasR, which is the regulator of las system (Figure 1B). Consistently with the dominant role of las system over $p q s$ and rhl systems, addition of BDSF also obviously inhibited $p q s$ and rhl systems (Figure 1C-D, Additional file 1: Figure S1), suggesting that the inhibitory activity of BDSF on the QS systems of $P$. aeruginosa may initial from the inhibition on las system. Recently, a DSF sensor, PA1396 was identified in P. aeruginosa [54]. To determine whether BDSF and DSF share the same sensor in P. aeruginosa, we then tested the BDSF effect on the virulence factor production mediated by QS systems in PA1396 deletion mutant. However, it was revealed that PA1396 is not BDSF sensor kinase, as disruption of PA1396 could not diminish the inhibitory effect of BDSF on the virulence factor production (Additional file 1: Figure S2). How does BDSF interfere with QS systems in P. aeruginosa still needs the further investigation.

T3SS is a key virulence determinant in a wide range of animal and plant pathogens and plays diverse roles in host-pathogen interactions. In $P$. aeruginosa, the transcriptional expression of effector genes of T3SS is coordinated by ExsA encoded by the exsCEBA operon. Our 
results showed that BDSF inhibited the expression of exs $C E B A$, which was confirmed by the results of semiquantitative RT-PCR analysis and western blotting analysis (Figure 3 ). It was recently reported that PsrA serves as a sensor for a long chain fatty acid (LCFA) to negatively modulate the expression of T3SS in $P$. aeruginosa [57]. However, exogenous addition of BDSF still showed a similar inhibition pattern on $p s r A$ deletion mutant, suggesting that BDSF may control T3SS through another sensor (Additional file 1: Figure S3). Moreover, the fact that T3SS is more sensitive to the exogenous addition of BDSF than the QS systems indicates that BDSF may interfere with QS systems and T3SS of $P$. aeruginosa through two independent signaling pathways. Besides the inhibitory effect on the QS systems and T3SS, our microarray analysis data suggests that BDSF also affects many other genes in $P$. aeruginosa (Data not shown). It was found that treatment with $250 \mu \mathrm{M}$ of BDSF up-regulated 66 genes more than 2 -fold, which is $1.38 \%$ to the total number of genes in genome. Meanwhile, a total of 120 genes were downregulated by more than 2 -fold (Data not shown). These genes are classified into the functional groups of metabolism, secretion, motility and cell wall, transcription regulation, protection, enzymes and carbon compound catabolism (Data not shown), suggesting that BDSF plays a global effect on $P$. aeruginosa.

Antibiotics have been used for a long time to treat the bacterial infection of $P$. aeruginosa, but resistance to antibiotics could be evolved during clinic treatment. Interestingly, recently studies found that some compounds successfully interfered with $N$-acyl homoserine lactone of $P$. aeruginosa and suppressed bacterial QS in lungs, finally caused accelerated lung bacterial clearance and reduced the severity of lung pathology [32]. Furthermore, some other compounds were identified to be the inhibitors of $P$. aeruginosa T3SS, treatment with these compounds caused the attenuated cytotoxicity of $P$. aeruginosa on mammalian cells [31]. Combined with our findings, it is suggested that interferences on the QS systems and T3SS can be specifically utilized as favorable therapeutic methods on $P$. aeruginosa infection $[31,32,34]$.

\section{Conclusions}

BDSF mediates the cross-talk between B. cenocepacia and $P$. aeruginosa by interfering with QS systems and T3SS of $P$. aeruginosa. Biological functions such as biofilm formation and virulence factor production of $P$. aeruginosa were inhibited by exogenous addition of BDSF. Furthermore, BDSF and some of its derivatives are also able to inhibit T3SS of $P$. aeruginosa at a micromolar level. Treatment with BDSF obviously reduced the virulence of $P$. aeruginosa in both in vitro and in vivo models.

\section{Methods}

\section{Bacterial strains and growth conditions}

The strains used in this work are listed in Table 1. P. aeruginosa PA14 strains were maintained in LuriaBertani (LB) broth at $37^{\circ} \mathrm{C}$. For analysis of T3SS, bacteria were grown in LB medium supplemented with the chelating reagent nitrilotiracetic acid (NTA) at a final concentration of $5 \mathrm{mM}$ NTA. The following antibiotics were supplemented when necessary: tetracycline, $100 \mu \mathrm{g} \mathrm{ml}^{-1}$; kanamycin, $50 \mu \mathrm{g} \mathrm{ml}^{-1}$; ampicillin, $200 \mu \mathrm{g} \mathrm{ml}^{-1}$. BDSF and its derivatives were synthesized as described previously [58]. They were added to the medium as indicated.

\section{Bacterial growth analysis}

Overnight bacterial cultures grown in LB broth was inoculated in the same medium to an $\mathrm{OD}_{600}$ of 0.025 in the absence and presence of BDSF. Three hundred microliters of inoculated culture were grown in each well at $37^{\circ} \mathrm{C}$ in a low intensity shaking model using the Bioscreen-C Automated Growth Curves Analysis System (OY Growth Curves AB Ltd, Finland).

\section{Construction of reporter strains and measurement of $\beta$-galactosidase activity}

The promoters of lasR, rhlR and pqsR were amplified using the primer pairs listed in Additional file 1: Table S2 with HindIII and EcoRI restriction sites attached. The resulting products were digested with HindIII and EcoRI, and ligated at the same enzyme sites in the vector pME2-lacZ. These constructs, verified by DNA sequencing, were introduced into $P$. aeruginosa by electroporation. Transconjugants were then selected on LB agar plates supplemented with tetracycline. For the construction of reporter strain of T3SS, the promoter of exsCEBA was amplified by PCR using the primer pairs listed in Additional file 1: Table S2, and cloned into the integration vector mini-CTX-lacZ [62]. The construct was introduced into E. coli $\mathrm{S} 17-1(\lambda$ pir) and then integrated into the chromosome of $P$. aeruginosa as described previously [63]. The engineered strain was then selected on the LB agar plates containing tetracycline and used as the T3SS reporter strain. Measurement of $\beta$-galactosidase activities was assayed following the methods as described previously [64]. Bacteria were grown at $37^{\circ} \mathrm{C}$ to an $\mathrm{OD}_{600}$ of about 1.5 , and the cells were harvested to measure the $\beta$-galactosidase activities.

\section{Biofilm formation assays}

Biofilm formation in 96-well polypropylene microtitre dishes was assayed as followed. Overnight bacterial cultures grown in LB broth was inoculated in the same medium to an $\mathrm{OD}_{600}$ of 0.01 in the absence and presence of BDSF signal at different concentrations as indicated. One hundred microliters of inoculated culture were grown in each well at 
Table 1 Bacterial strains and plasmids used in this study

\begin{tabular}{|c|c|c|}
\hline Strain or plasmid & Phenotypes and/or characteristics & Reference or source \\
\hline \multicolumn{3}{|l|}{ B. cenocepacia } \\
\hline WT & J2315 Wild type strain, Genomovars III of B. cepacia complex & ATCC \\
\hline d0581 & BDSF-minus mutant derived from J2315 with Bcam0581 being deleted & 35 \\
\hline d0581(0581) & Mutant d0581 harboring the expression construct pMLS7-0581 & 35 \\
\hline \multicolumn{3}{|l|}{ P. aeruginosa } \\
\hline PA14 & Clinical isolate & Laboratory collection \\
\hline PA14(PCZ) & PA14 harboring the T3SS reporter construct mini-CTX-lacZ with the exsCEBA promoter fused to lacZ & This study \\
\hline PA14 (PlasR-lacZ) & PA14 harboring the reporter construct pME2PlasR-lacZ & This study \\
\hline PA14 (PpqsR-lacZ) & PA14 harboring the reporter construct pME2PpqsR-lacZ & This study \\
\hline PA14 (PrhlR-lacZ) & PA14 harboring the reporter construct pME2PrhIR-lacZ & This study \\
\hline dpsrA (PCZ) & $\begin{array}{l}\text { psrA deletion mutant harboring the T3SS reporter construct mini-CTX-lacZ with the exsCEBA } \\
\text { promoter fused to lacZ }\end{array}$ & This study \\
\hline dPA1396 & PA1396 deletion mutant derived from PA14 & This study \\
\hline \multicolumn{3}{|l|}{ E.coli } \\
\hline S17-1 & Res $^{-}$pro mod ${ }^{+}$integrated copy of RP4, mob ${ }^{+}$, used for incorporating & Laboratory collection \\
\hline CV026 & The biosensor strain of $\mathrm{C} 4-\mathrm{HSL}$ & Laboratory collection \\
\hline \multicolumn{3}{|l|}{$\begin{array}{l}\text { Agrobacterium } \\
\text { tumefaciens }\end{array}$} \\
\hline CF11 & $\mathrm{AHL}$ reporter strain & $\begin{array}{l}\text { Lab of Stephen K. } \\
\text { Farrand }\end{array}$ \\
\hline \multicolumn{3}{|l|}{ Plasmid } \\
\hline PCZ & mini-CTX-lacZ with the exsCEBA promoter fused to lacZ & 62 \\
\hline PlasR-lacZ & pME2-lacZ containing promoter of las $R$ & This study \\
\hline PpqsR-lacZ & pME2-lacZ containing promoter of pqs $R$ & This study \\
\hline PrhIR-lacZ & pME2-lacZ containing promoter of $r h / R$ & This study \\
\hline
\end{tabular}

$37^{\circ} \mathrm{C}$ with shaking at $150 \mathrm{rpm}$ for $18 \mathrm{~h}$. The cultures were removed and $200 \mu \mathrm{l}$ of $1 \%$ crystal violet (w/v) was added. Following staining at room temperature for $15 \mathrm{~min}$, the dye was removed and the wells were rinsed three times with water. For quantification of the attached bacterial cells, the stained wells were decolorized with $200 \mu \mathrm{l}$ of $95 \%$ ethanol. The quantity of crystal violet was determined by measuring the absorbance at $595 \mathrm{~nm}$.

\section{Proteolytic activity assay}

Protease activity was quantified following the previously described method with minor modifications [65]. Briefly, bacteria were cultured at $37^{\circ} \mathrm{C}$ for about 12 hours. After measuring the optical density at $600 \mathrm{~nm}$, cultures were centrifuged at 13,000 $\mathrm{rpm}$ for $5 \mathrm{~min}$ and the supernatants were taken out and filtered through a $0.2 \mu \mathrm{m}$ pore size cellulose-acetate filter. One hundred microliter of supernatants were incubated at $30^{\circ} \mathrm{C}$ with equal volume of azocasein dissolved in proteolytic buffer B $(5 \mathrm{mg} / \mathrm{ml})$ for $30 \mathrm{~min}$. The reaction was stopped by addition of $400 \mu \mathrm{l}$ of $10 \%(\mathrm{w} / \mathrm{v})$ TCA buffer. After incubation for $2 \mathrm{~min}$ at room temperature, the mixture was centrifuged at $13,000 \mathrm{rpm}$ for $1 \mathrm{~min}$ to remove the remaining azocasein.
Supernatants were taken out and mixed with $700 \mu \mathrm{l}$ of $525 \mathrm{mM} \mathrm{NaOH}$. Absorbance of the azopeptide supernatant was measured at the wavelength of $442 \mathrm{~nm}$. Protease activity was obtained after normalization the absorbance against the corresponding cell density.

\section{Pyocyanin assay}

After measuring the absorbance at $600 \mathrm{~nm}$, the supernatants of bacterial cultures were collected for extraction of pyocyanin with the method described previously [66]. Briefly, cultures were centrifuged at 13,000 rpm for $1 \mathrm{~min}$ and $1.5 \mathrm{ml}$ supernatants were collected and extracted with double volume chloroform with vigorous shaking at room temperature for $30 \mathrm{~min}$. The solvent phase was transferred to a new tube containing $1 \mathrm{ml}$ of $1 \mathrm{~N} \mathrm{HCl}$. The mixture was shaken gently to transfer pyocyanin to aqueous phase. The quantity of pyocyanin was determined by measurement of absorbance at $520 \mathrm{~nm}$ and normalization against the cell density.

\section{RNA extraction and RT-PCR analysis}

$P$. aeruginosa was grown in LB medium supplemented with NTA till $\mathrm{OD}_{600}$ of 1.5. Total RNA was isolated 
using the RNeasy mini kit (Qiagen) according to the manufacturer's instructions. The concentration and purity of RNA were determined by agarose gel electrophoresis and spectrometry. Reverse transcription (RT-PCR) analysis was performed using the One-step RT-PCR kit according to the manufacturer's instructions (Qiagen).

\section{Protein isolation and western blotting analysis}

Bacteria were cultured in LB medium supplemented with NTA to an $\mathrm{OD}_{600}$ of $1.5,10 \mathrm{ml}$ of each bacterial culture were collected and centrifuged. The supernatants were then filtered with $0.2 \mu \mathrm{m}$ syringe filter and precipitated with trichloroacetic acid (TCA) at a final concentration of $10 \%$. The precipitates were pelleted by centrifugation, washed twice with acetone, dried, and re-suspended in SDS sampling buffer. The protein samples were denatured by boiling for $5 \mathrm{~min}$ and separated by 10\% SDS-PAGE. Western blot analysis was performed following the standard protocols [67].

\section{Cytotoxicity assays in HeLa cell model}

BDSF effect on the cytotoxicity of $P$. aeruginosa 14 was assayed by using HeLa cells. HeLa cells were seeded in 24-well tissue culture plates containing Dulbecco's Modified Eagle Medium (DMEM) and allowed to grow at $37^{\circ} \mathrm{C}$ in $\mathrm{CO}_{2}$ for about 18 hours to obtain $80-90 \%$ monolayer confluency $\left(5.0 \times 10^{5}\right.$ cells/well). Culture supernatants were removed and the monolayer was washed once with PBS buffer. Fresh bacterial cells were diluted in DMEM to a concentration about $5 \times 10^{7} \mathrm{CFU}$ per ml. Thereafter, $0.5 \mathrm{ml}$ of bacteria dilutions in the absence and presence of BDSF were applied to the HeLa cell monolayers at a multiplicity of infection (MOI) about 50. Cytotoxicity was determined by measuring the release of the cytosolic enzyme lactate dehydrogenase (LDH) into supernatants using the cytotoxicity detection kit (Roche).

\section{Virulence assays using a zebrafish infection model}

$P$. aeruginosa PA14 virulence was tested by infecting 6-month-old zebrafish (Danio rerio). Firstly, bacterial cultures were grown to an $\mathrm{OD}_{600}$ of 1.0, and washed with phosphate-buffered saline (PBS; $\mathrm{pH}$ 7.3), then dissolved in PBS buffer to $5.0 \times 10^{9} \mathrm{cfu} \mathrm{ml}^{-1}$ in the presence of $100 \mu \mathrm{M}$ BDSF or equal volume methanol, respectively. To this end, $30 \mu \mathrm{l}$ bacterial cultures were injected intraperitoneally into each fish by using a $1-\mathrm{ml}$ tuberculin syringe attached to a 30.5-gauge Precision Glide needle (Becton Dickinson). The experiment was repeated three times, each time using 10 fish of similar body mass per treatment. Mortality was scored daily, and dead fish was removed immediately.

Zebrafish experiments were conducted in accordance with the guidelines of Institutional Biosafety Committee and Institutional Ethics committee (IBC-IEC) of
Institute of Molecular and Cell Biology (IMCB) of Singapore. Animal infection experiments were approved by IBC-ITE of IMCB.

\section{Additional file}

Additional file 1: Figure S1. Inhibition of exogenous addition of BDSF on the production of 3-OXO-C12-HSL (A), PQS (B) and C4-HSL (C) of P. aeruginosa PA14. Figure S2. Inhibitory effect of BDSF on the production of extracellular protease (A) and pyocyanin (B) of PA1396 deletion mutant of $P$. aeruginosa PA14. The data are the means of three repeats and error bars indicate the standard deviations. Figure S3. Inhibitory effect of BDSF on T3SS of psrA deletion mutant of $P$. aeruginosa PA14, as determined by using PexsCEBAlacZ fusion reporter strain. The data are the means of three repeats and error bars indicate the standard deviations. Table S1. Chemical structures of BDSF and its derivatives. Table S2. PCR primers used in this study.

Competing interest

The authors declare that they have no competing interest.

Authors' contributions

Experiments were carried out by YD, BC, SC, AL. Data analysis was finished by $Y D$ and LHZ. The study was designed by YD and LHZ, who also drafted the manuscript. All authors read and approved the final manuscript.

\section{Acknowledgements}

The funding for this work was provided by the Biomedical Research Council, Agency of Science, Technology, and Research (A*Star), Singapore.

Received: 1 August 2013 Accepted: 15 October 2013

Published: 18 October 2013

\section{References}

1. Whitehead NA, Barnard AM, Slater H, Simpson NJ, Salmond GP: Quorumsensing in Gram-negative bacteria. FEMS Microbiol Rev 2001, 25:365-404.

2. Fuqua C, Greenberg EP: Listening in on bacteria: acyl-homoserine lactone signalling. Nat Rev Mol Cell Biol 2002, 3:685-695.

3. He YW, Zhang LH: Quorum sensing and virulence regulation in Xanthomonas campestris. FEMS Microbiol Rev 2008, 32:842-857.

4. Deng Y, Wu J, Tao F, Zhang LH: Listening to a new language: DSF-based quorum sensing in gram-negative. Chem Rev 2011, 111:160-173.

5. Bodey GP, Bolivar R, Fainstein V, Jadeja L: Infections caused by Pseudomonas aeruginosa. Rev Infect Dis 1983, 5:279-313.

6. Richards MJ, Edwards JR, Culver DH, Gaynes RP: Nosocomial infections in combined medical-surgical intensive care units in the United States. Infect Control Hosp Epidemiol 2000, 21:510-515.

7. Wagner VE, Li LL, Isabella VM, Iglewski BH: Analysis of the hierarchy of quorum-sensing regulation in Pseudomonas aeruginosa. Anal Bioanal Chem 2007, 387:469-479.

8. Feltman H, Schulert G, Khan S, Jain M, Peterson L, Hauser AR: Prevalence of type III secretion genes in clinical and environmental isolates of Pseudomonas aeruginosa. Microbiol 2001, 147:2659-2669.

9. Moss J, Ehrmantraut ME, Banwart BD, Frank DW, Barbieri JT: Sera from adult patients with cystic fibrosis contain antibodies to Pseudomonas aeruginosa type III apparatus. Infect Immun 2001, 69:1185-1188.

10. Sato H, Frank DW: ExoU is a potent intracellular phospholipase. Mol Microbiol 2004, 53:1279-1290.

11. Yahr TL, Goranson J, Frank DW: Exoenzyme S of Pseudomonas aeruginosa is secreted by a type III pathway. Mol Microbio/ 1996, 22:991-1003.

12. Yahr TL, Vallis AJ, Hancock MK, Barbieri JT, Frank DW: ExoY, an adenylate cyclase secreted by the Pseudomonas aeruginosa type III system. Proc Natl Acad Sci USA 1998, 95:13899-13904.

13. Yahr $\mathrm{TL}$, Wolfgang MC: Transcriptional regulation of the Pseudomonas aeruginosa type III secretion system. Mol Microbiol 2006, 62:631-640.

14. Hueck CJ: Type III protein secretion systems in bacterial pathogens of animals and plants. Microbiol Mol Biol Rev 1998, 62:379-433.

15. Pallen MJ, Beatson SA, Bailey CM: Bioinformatics, genomics and evolution of non-flagellar type-III secretion systems: a Darwinian perspective. FEMS Microbiol Rev 2005, 29:201-229. 
16. Waters CM, Wu JT, Ramsey ME, Harris RC, Bassler BL: Control of the Type 3 Secretion system in Vibrio harveyi by quorum sensing through repression of ExsA. Appl Environ Microbiol 2010, 76:4996-5004.

17. Sperandio V, Mellies JL, Nguyen W, Shin S, Kaper JB: Quorum sensing controls expression of the type III secretion gene transcription and protein secretion in enterohemorrhagic and enteropathogenic Escherichia coli. Proc Natl Acad Sci USA 1999, 96:15196-15201.

18. Zhang $\mathrm{LH}$, Dong $\mathrm{YH}$ : Quorum sensing and signal interference: diverse implications. Mol Microbiol 2004, 53:1563-1571.

19. Dong $\mathrm{YH}$, Zhang LH: Quorum sensing and quorum-quenching enzymes. J Microbiol 2005, 43:101-109.

20. Hogan DA, Vik A, Kolter R: A Pseudomonas aeruginosa quorum-sensing molecule influences Candida albicans morphology. Mol Microbiol 2004 54:1212-1223.

21. Ishida T, Ikeda T, Takiguchi N, Kuroda A, Ohtake H, Kato J: Inhibition of quorum sensing in Pseudomonas aeruginosa by $\mathrm{N}$-acyl cyclopentylamides. Appl Environ Microbiol 2007, 73:3183-3188.

22. Kim C, Kim J, Park HY, Park HJ, Lee JH, Kim CK, Yoon J: Furanone derivatives as quorum-sensing antagonists of Pseudomonas aeruginosa. Appl Microbiol Biotechnol 2008, 80:37-47.

23. Smyth AR, Cifelli PM, Ortori CA, Righetti K, Lewis S, Erskine P, Holland ED, Givskov M, Williams P, Cámara M, Barrett DA, Knox A: Garlic as an inhibitor of Pseudomonas aeruginosa quorum sensing in cystic fibrosis-a pilot randomized controlled trial. Pediatr Pulmonol 2010, 45:356-362.

24. Dobretsov S, Teplitski M, Alagely A, Gunasekera SP, Paul VJ: Malyngolide from the cyanobacterium Lyngbya majuscula interferes with quorum sensing circuitry. Environ Microbiol Rep 2010, 2:739-744.

25. Jakobsen TH, Bragason SK, Phipps RK, Christensen LD, van Gennip M Alhede M, Skindersoe M, Larsen TO, Høiby N, Bjarnsholt T, Givskov M: Food as a source for quorum sensing inhibitors: iberin from horseradish revealed as a quorum sensing inhibitor of Pseudomonas aeruginosa Appl Environ Microbiol 2012, 78:2410-2421

26. Bobadilla Fazzini RA, Skindersoe ME, Bielecki P, Puchałka J, Givskov M, Santos VA M s: Protoanemonin: a natural quorum sensing inhibitor that selectively activates iron starvation response. Environ Microbiol 2013 15:111-120.

27. Majik MS, Naik D, Bhat C, Tilve S, Tilvi S, D'Souza L: Synthesis of (R)norbgugaine and its potential as quorum sensing inhibitor against Pseudomonas aeruginosa. Bioorg Med Chem Lett 2013, 23:2353-2356.

28. Norizan SN, Yin WF, Chan KG: Caffeine as a potential quorum sensing inhibitor. Sensors (Basel) 2013, 13:5117-5129.

29. Wang J, Dong Y, Zhou T, Liu X, Deng Y, Wang C, Lee J, Zhang LH: Pseudomonas aeruginosa cytotoxicity is attenuated at high cell density and associated with the accumulation of phenylacetic acid. PLOS One 2013, 8:e60187.

30. Grier MC, Garrity-Ryan LK, Bartlett VJ, Klausner KA, Donovan PJ, Dudley C, Alekshun MN, Tanaka SK, Draper MP, Levy SB, Kim OK: N-Hydroxybenzimidazole inhibitors of ExsA MAR transcription factor in Pseudomonas aeruginosa: In vitro anti-virulence activity and metabolic stability. Bioorg Med Chem Lett 2010, 20:3380-3383.

31. Aiello D, Williams JD, Majgier-Baranowska H, Patel I, Peet NP, Huang J, Lory S, Bowlin TL, Moir DT: Discovery and characterization of inhibitors of Pseudomonas aeruginosa type III secretion. Antimicrob Agents Chemother 2010, 54:1988-1999.

32. Wu H, Song Z, Hentzer M, Andersen JB, Molin S, Givskov M, Høiby N: Synthetic furanones inhibit quorum-sensing and enhance bacterial clearance in Pseudomonas aeruginosa lung infection in mice. J Antimicrobial Chemotherapy 2004, 53:1054-1061.

33. Keyser P, Elofsson M, Rosell S, Wolf-Watz H: Virulence blockers as alternatives to antibiotics: type III secretion inhibitors against Gram-negative bacteria. J Intern Med 2008, 264:17-29.

34. Hentzer $M$, Givskov M: Pharmacological inhibition of quorum sensing for the treatment of chronic bacterial infections. J Clin Invest 2003, 112:1300-1307.

35. Boon C, Deng Y, Wang LH, He Y, Xu JL, Fan Y, Pan SQ, Zhang LH: A novel DSF-like signal from Burkholderia cenocepacia interferes with Candida albicans morphological transition. ISME J 2008, 2:27-36.

36. Deng $Y$, Boon C, Eberl L, Zhang LH: Differential modulation of Burkholderia cenocepacia virulence and energy metabolism by quorum sensing signal BDSF and its synthase. J Bacteriol 2009, 191:7270-7278.

37. Deng Y, Wu J, Eberl L, Zhang LH: Structural and functional characterization of diffusible signal factor family quorum-sensing signals produced by members of the Burkholderia cepacia complex. App/ Environ Microbiol 2010, 76:4675-4683.

38. Deng Y, Schmid N, Wang C, Wang J, Pessi G, Wu D, Lee J, Aguilar C, Ahrens $\mathrm{CH}$, Chang C, Song H, Eberl L, Zhang LH: Cis-2-dodecenoic acid receptor RpfR links quorum-sensing signal perception with regulation of virulence through cyclic dimeric guanosine monophosphate. Proc Natl Acad Sci USA 2012, 109:15479-15484.

39. Schmid N, Pessi G, Deng Y, Aguilar C, Carlier AL, Grunau A, Omasits U, Zhang LH, Ahrens CH, Eberl L: The AHL- and BDSF-dependent quorum sensing systems control specific and overlapping sets of genes in Burkholderia cenocepacia H111. PLoS One 2012, 7(11):e49966.

40. Deng Y, Lim A, Wang J, Zhou T, Chen S, Lee J, Dong YH, Zhang LH: Cis-2-dodecenoic acid quorum sensing system modulates $\mathrm{N}$-acyl homoserine lactone production through RpfR and cyclic di-GMP turnover in Burkholderia cenocepacia. BMC Microbiol 2013, 13:148.

41. McCarthy Y, Yang L, Twomey KB, Sass A, Tolker-Nielsen T, Mahenthiralingam E, Dow JM, Ryan RP: A sensor kinase recognizing the cell-cell signal BDSF (cis-2-dodecenoic acid) regulates virulence in Burkholderia cenocepacia. Mol Microbiol 2010, 77:1220-1236.

42. Twomey KB, O'Connell OJ, McCarthy Y, Dow JM, O'Toole GA, Plant BJ, Ryan RP: Bacterial cis-2-unsaturated fatty acids found in the cystic fibrosis airway modulate virulence and persistence of Pseudomonas aeruginosa. ISMEJ 2012, 6:939-950.

43. Schuster M, Lostroh CP, Ogi T, Greenberg EP: Identification, timing, and signal specificity of Pseudomonas aeruginosa quorum-controlled genes: a transcriptome analysis. J Bacteriol 2003, 185:2066-2079.

44. Wagner VE, Bushnell D, Passador L, Brooks Al, Iglewski BH: Microarray analysis of Pseudomonas aeruginosa quorum-sensing regulons: effects of growth phase and environment. J Bacterio/ 2003, 185:2080-2095.

45. Latifi A, Foglino M, Tanaka K, Williams P, Lazdunski A: A hierarchical quorum-sensing cascade in Pseudomonas aeruginosa links the transcriptional activators LasR and RhIR (VsmR) to expression of the stationary-phase sigma factor RpoS. Mol Microbiol 1996, 21:1137-1146.

46. Pesci EC, Pearson JP, Seed PC, Iglewski BH: Regulation of las and rhl quorum sensing in Pseudomonas aeruginosa. J Bacteriol 1997, 179:3127-3232.

47. Pesci EC, Milbank JB, Pearson JP, McKnight S, Kende AS, Greenberg EP, Iglewski BH: Quinolone signaling in the cell-to-cell communication system of Pseudomonas aeruginosa. Proc Natl Acad Sci USA 1999 96:11229-11234

48. McKnight SL, Iglewski BH, Pesci EC: The Pseudomonas Quinolone Signal Regulates $r$ l Quorum Sensing in Pseudomonas aeruginosa. J Bacteriol 2000, 182:2702-2708.

49. Lee J, Wu J, Deng Y, Wang J, Wang C, Wang J, Chang C, Dong Y, Williams P, Zhang LH: A cell-cell communication signal integrates quorum sensing and stress response. Nat Chem Biol 2013, 9:339-343.

50. Holder IA, Neely AN: The role of proteases in Pseudomona infections in burns: a current hypothesis. Antibiot Chemother 1991, 44:99-105.

51. Mahajan-Miklos S, Tan MW, Rahme LG, Ausubel FM: Molecular mechanisms of bacterial virulence elucidated using a Pseudomonas aeruginosa Caenorhabdititis elegans pathogenesis model. Cell 1999, 96:47-56.

52. Rahme LG, Ausubel FM, Cao H, Drenkard E, Goumnerov BC, Lau GW, Mahajan-Miklos S, Plotnikova J, Tan MW, Tsongalis J, Walendziewicz CL, Tompkins RG: Plants and animals share functionally common bacterial virulence factors. Proc Natl Acad Sci U S A 2000, 97:8815-8821.

53. Ran H, Hassett DJ, Lau GW: Human targets of Pseudomonas aeruginosa pyocyanin. Proc Natl Acad Sci U S A 2003, 100:14315-14320.

54. Ryan RP, Fouhy Y, Garcia BF, Watt SA, Niehaus K, Yang L, Tolker-Nielsen T, Dow JM: Interspecies signalling via the Stenotrophomonas maltophilia diffusible signal factor influences biofilm formation and polymyxin tolerance in Pseudomonas aeruginosa. Mol Microbiol 2008, 68:75-86

55. Frank DW: The exoenzyme $\mathrm{S}$ regulon of Pseudomonas aeruginosa. Mol Microbiol 1997, 26:621-629.

56. Hovey AK, Frank DW: Analyses of the DNA-binding and transcriptional activation properties of ExsA, the transcriptional activator of the Pseudomonas aeruginosa exoenzyme S regulon. J Bacteriol 1995, 177:4427-4436.

57. Kang $Y$, Lunin $W$, Skarina $T$, Savchenko A, Schurr MJ, Hoang TT: The longchain fatty acid sensor, PsrA, modulates the expression of rpoS and the type III secretion exsCEBA operon in Pseudomonas aeruginosa. Mol Microbiol 2009, 73:120-136. 
58. Wang $L H$, He Y, Gao Y, Wu JE, Dong YH, He C, Wang SX, Weng LX, Xu JL, Tay L, Fang RX, Zhang LH: A bacterial cell-cell communication signal with cross-kingdom structural analogues. Mol Microbiol 2004, 51:903-912.

59. Clatworthy AE, Lee JS, Leibman M, Kostun Z, Davidson AJ, Hung DH: Pseudomonas aeruginosa infection of zebrafish involves both host and pathogen determinants. Infect Immun 2009, 77:1293-1303.

60. Mckenney D, Brown KE, Allison DG: Influence of Pseudomonas aeruginosa Exoproducts on Virulence Factor Production in Burkholderia cepacia: Evidence of Interspecies Communication. J Bacteriol 1995, 177:6989-6992.

61. Riedel K, Hentzer M, Geisenberger O, Huber B, Steidle A, Wu H, Høiby N, Givskov M, Molin S, Eberl L: N-Acylhomoserine-lactone-mediated communication between Pseudomonas aeruginosa and Burkholderia cepacia in mixed biofilms. Microbiology 2001, 147:3249-3262.

62. Zhou L, Wang J, Zhang LH: Modulation of Bacterial Type III Secretion System by a Spermidine Transporter Dependent Signaling Pathway. PLOS ONE 2007, 2:e1291.

63. Hoang TT, Kutchma AJ, Becher A, Schweizer HP: Integration-proficient plasmids for Pseudomonas aeruginosa: site-specific integration and use for engineering of reporter and expression strains. Plasmid 2000, 43:59-72.

64. Miller JH: A short course in bacterial genetics: A Laboratory Manual and Handbook for Escherichia Coli and Related Bacteria. Cold Spring Harbor, New York: Cold Spring Harbor Laboratory Press; 1992.

65. Safarík I: A modified procedure for the preparation of insoluble chromogenic substrates for the determination of proteolytic activity. J Biochem Biophys Methods 1987, 14:355-357.

66. Essar DW, Eberly L, Hadero A, Crawford IP: Identification and characterization of genes for a second anthranilate synthase in Pseudomonas aeruginosa: interchangeability of the two anthranilate synthases and evolutionary implications. J Bacteriol 1990, 172:884-900.

67. Sambrook J, Fritsch EF, Maniatis T: Molecular Cloning: A Laboratory Manual. Cold Spring Harbor, New York: Cold Spring Harbor Laboratory Press; 1987.

doi:10.1186/1471-2180-13-231

Cite this article as: Deng et al: Cis-2-dodecenoic acid signal modulates virulence of Pseudomonas aeruginosa through interference with quorum sensing systems and T3SS. BMC Microbiology 2013 13:231.

\section{Submit your next manuscript to BioMed Central and take full advantage of:}

- Convenient online submission

- Thorough peer review

- No space constraints or color figure charges

- Immediate publication on acceptance

- Inclusion in PubMed, CAS, Scopus and Google Scholar

- Research which is freely available for redistribution 